\title{
Influence of the Plasma Opacity in the Calculations of IR Emission Due to Dielectronic Recombination of Mg II
}

\author{
Alicia Cruzado ${ }^{1}$ and Adela E. Ringuelet ${ }^{2}$ \\ Facultad de Ciencias Astronómicas y Geofísicas, Universidad Nacional \\ de La Plata, Paseo del Bosque s/n, 1900-La Plata, Argentina
}

\begin{abstract}
In an earlier work (Cruzado, Di Rocco \& Ringuelet, 1998), we showed that the dielectronic recombination of $\mathrm{Mg}$ II atoms contributes strongly to the infrared flux. In the present paper we aim at investigating whether the approximations made before could have biased our conclusions.
\end{abstract}

\section{Introduction}

An envelope surrounding the star distinguishes Be stars from normal B stars; all observed spectral features that characterize a Be spectrum, must originate in such envelope. As a consequence, in order to explain the observations, all possible physical processes taking place in that envelope ought to be analyzed. At present we are studying the dielectronic recombination (hereafter DR) of metals, a phenomenon that must be taken into account (Burgess \& Seaton, 1964) in low density plasmas. As a first step $\mathrm{Mg}$ II has been selected first because of the astrophysical significance of this element and of its simple atomic structure. In a previous paper (Cruzado, Di Rocco \& Ringuelet, 1998, hereafter Paper I) we were able to show how the DR of $\mathrm{Mg}$ II atoms in the envelope of early type stars, contributes to the infrared flux. In that paper we considered $\mathrm{Mg}$ II atoms in the ground state capturing free electrons and, as a consequence, producing $\mathrm{Mg} \mathrm{I}$ atoms in autoionizing levels above the first ionization limit. Since those autoionizing levels are unstable states, autoionization or transitions to levels lying below the first ionization limit could occur; if the second option would be the case, cascading to lower bound levels will give rise to emission lines. In order to evaluate the contribution of this atomic process to the stellar flux, we calculated the energy emitted in those lines. In order to do so, we solved the statistical equilibrium equations and computed transitions probabilities by applying the Hartree-Fock methodology. The results of Paper I were obtained by accepting specific approximations, the significance of which we will analyze in this work, namely, a) the influence on the results of the shift of the calculated energy levels relative to the observed ones and, b) the plasma opacity.

\footnotetext{
${ }^{1}$ Fellow of the University of La Plata

${ }^{1}$ Member of the Carrera del Investigador Científico, CONICET, Argentina
} 


\section{Influence of the energy level shift on the results}

The different atomic parameters were calculated by using the Hartree-Fock methodology by means of Cowan's code. The locations of the energy levels obtained this way are shifted relative to the observed ones. Cowan's code allows us to adjust the values of the theoretical parameters to produce calculated energy levels with the best possible agreement with the experimental ones. We achieved this with a group of configurations and, when the final values of the parameters had been obtained, we improved the calculation of the transition probabilities and compute the emitted energy again. The flux excesses obtained by using the theoretical parameters differ less than $20 \%$ from the ones obtained by using adjusted parameters.

\section{Influence of the plasma opacity}

In Paper I we had assumed the DR of the $\mathrm{Mg}$ II atoms taking place in a completely transparent region. Now we will consider different possible sources of opacity and their influence on the emitted flux.

In considering the continuum opacity of the envelope, we have taken into account the bound-free and the free-free transitions in hydrogen atoms and negative ions of hydrogen, together with Rayleigh Thomson scattering. By using the expressions given by Kurucz (1970), we calculated the absorption coefficients for different wavelengths, temperatures and electronic densities.

If we illustrate the behavior of $\tau$ when modifying the electronic temperature, we will see how it increases when the temperature decreases. The rise in the opacity curve starts at $T=T_{c}$ and reaches the value $1(\tau=1)$ at $T=T_{0}$. At fixed value of the electronic density, the values of $T_{c}$ and $T_{0}$ depend on the wavelength; for an electronic density of $10^{9} \mathrm{~cm}^{-3}, 3600 \mathrm{~K} \leq T_{0} \leq 4300 \mathrm{~K}$, provided $0.3 \mu \leq \lambda \leq 100 \mu$. If the electronic density decreases, the $T_{0}$ values shift towards lower temperatures. But, for $\mathrm{T}$ values lower than $3000 \mathrm{~K}$ the $\mathrm{DR}$ of the $\mathrm{Mg}$ II atoms is no longer important because of the location of the first autoionizing level of the $\mathrm{Mg}$ I atom: for such low temperature there will be not enough free electrons with sufficient energy so as to reach the autoionizing levels.

It is apparent that the opacity increase with the electronic density. For high values of the density, the $\mathrm{DR}$ of $\mathrm{Mg}$ II atoms, as a process that contributes to the emitted flux, will not be effective because when the density goes up we will have more and more bound levels being depopulated by collisionally induced processes before a radiative decay.

The behavior of the opacity with wavelength is shown in Fig. 1; we observe how it increase at both sides of a minimum value of $\lambda=\lambda_{m}$. The value of $\lambda_{m}$ depends on the temperature if the electronic density is set; thus, for an electronic density of $10^{9} \mathrm{~cm}^{-3}$ and $4500 \mathrm{~K} \geq T \geq 3000 \mathrm{~K}, 1.6 \mu \leq \lambda_{m} \leq 1.9 \mu$, the minimum being very pronounced for $T \leq 4500 \mathrm{~K}$. This interval of values of $\lambda_{m}$ shifts towards longer values, if the electronic density decreases.

In order to estimate how the emitted flux may be influenced by the continuum opacity, we have introduced an $e^{-\tau_{c}}$ factor, $\tau_{c}$ being calculated through the expression

$$
\tau_{c}=\left(\alpha_{B F H}+\alpha_{F F H}+\alpha_{B F H^{-}}+\alpha_{F F H^{-}}+\alpha_{R S}+\alpha_{T S}\right) L
$$




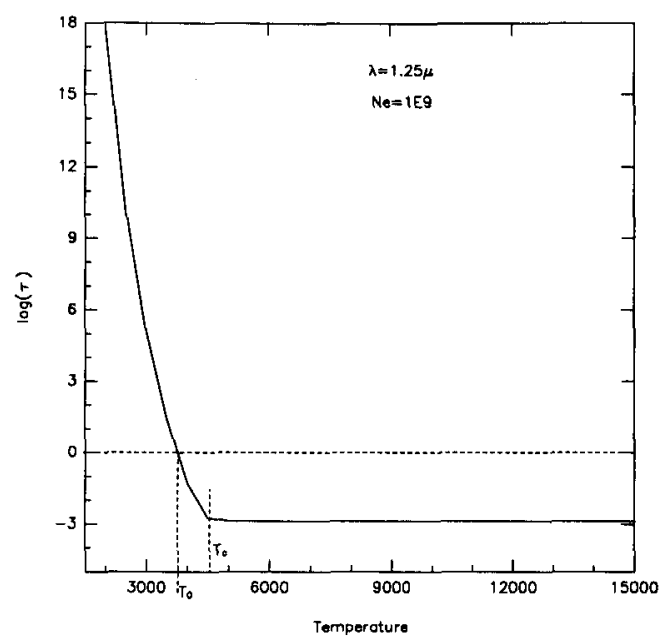

Figure 1. Behaviour of the opacity with the wavelength, for a set value of the electron density and two values of the temperature.

where the $\alpha$ 's represent the different absorption coefficients, and, L, the geometric thickness of the region whose opacity is calculated. We evaluated $\tau_{c}$ for different values of the electronic density and temperature, setting $L$ at $7 R_{*}$. For an electron density of $10^{9} \mathrm{~cm}^{-3}$, no excess is observed at $T \leq 3600 \mathrm{~K}$ : the plasma is actually opaque for the flux originated in the DR of $\overline{M g}$ II at such low temperature, while at $T \geq 4300 \mathrm{~K}$ the flux remains unchanged, the plasma being completely transparent. It principally decreases at very short and at very large wavelengths for intermediate temperature values (Fig. 2). For an electron density of $10^{10} \mathrm{~cm}^{-3}$, the temperature values at which the plasma behaves as completely opaque or completely transparent, for every wavelength values, shift to $4200 \mathrm{~K}$ and to $9300 \mathrm{~K}$, respectively. At $T>5000 \mathrm{~K}$, however, the flux is only affected for $\lambda>12 \mu$.

\section{Conclusions}

We have suggested that the DR of Mg II may contribute significantly to the IR excess in Be stars (Paper I). In a second step, we have verified that improvements in the determination of atomic energy levels do not produce any considerable modification in the results when we compare them with broad-band observations.

The physical conditions in the envelope will determine how important the plasma opacity is. Specifically, the temperature and density distributions with the distance from the central star, that is to say, the atmospheric model that is adopted, will be decisive.

Let us take into account that we have considered, as a zero-order approximation, $\mathrm{DR}$ of $\mathrm{Mg}$ II taking place in a homogeneous region with a constant 


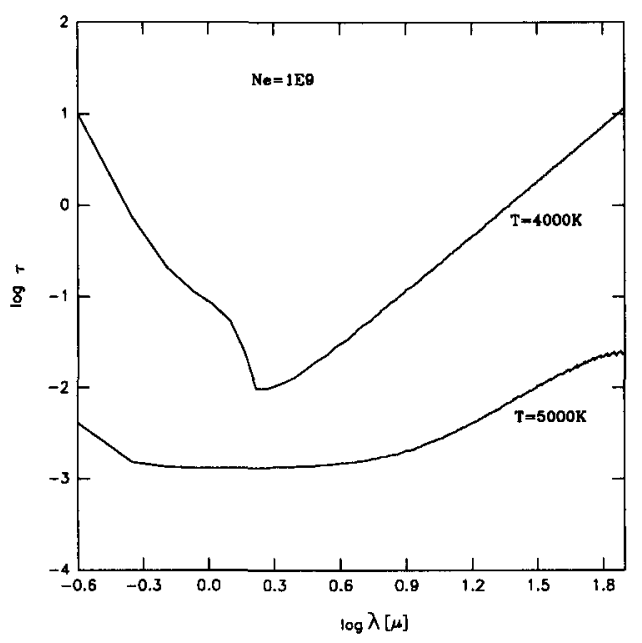

Figure 2. Influence of the continuum opacity on the emitted flux.

temperature and a constant density. The electronic density and temperature values at which the DR of $\mathrm{Mg}$ II is important suggest that this region will be located quite far from the star. In that region the plasma opacity is not significant for most wavelength values; there is a small temperature interval for which the DR of $\mathrm{Mg}$ II is important and the opacity reaches large values, but, only for very short and very large wavelength values (the effect of the opacity is to make more evident the two hills that the flux curve shows). The opacity in the inner layer, due to the processes considered in the present work, will not affect the emitted flux that arise from the DR of $\mathrm{Mg}$ II; the photospheric flux will be affected by the opacity in the inner layers where the electronic density is high, if the temperature is sufficiently low. If the envelope is very extended, the value of the opacity could be important in the outer layers, where the temperature would be lower.

Since the DR of Mg II actually occurs in different regions with different temperatures and different densities rather than in an homogeneous region with constant temperature and constant density, it is our purpose to adopt in a next paper a more specific atmospheric model and insert the process in a suitable form.

\section{References}

Burgess, A., Seaton, M.J. 1964, MNRAS 127, 355.

Cruzado, A., Di Rocco, H.O., Ringuelet, A. 1998, ApJ 503, 902-915 (Paper I).

Kurucz, R.L. 1970, ATLAS SAO Special Report 309. 